\title{
High body mass index is associated with an increased overall survival in rectal cancer
}

\author{
Karolina Juszczyk ${ }^{1}$, Sharlyn Kang ${ }^{2,3}$, Soni Putnis ${ }^{1}$, Robert Winn ${ }^{1}$, James Chen ${ }^{2}$, Morteza Aghmesheh ${ }^{3,4}$, \\ Glaucia Fylyk ${ }^{5}$, Daniel Brungs ${ }^{3,4}$ \\ ${ }^{1}$ Division of Surgery, Colorectal Unit, Wollongong Hospital, NSW, Australia; ${ }^{2}$ Department of Radiation Oncology, Illawarra Cancer Care Centre, \\ Wollongong Hospital, NSW, Australia; ${ }^{3}$ Illawarra Health and Medical Research Institute, University of Wollongong, NSW, Australia; ${ }^{4}$ Department \\ of Medical Oncology, Illawarra Cancer Care Centre, Wollongong Hospital, NSW, Australia; ${ }^{5}$ Department of Radiation Oncology, Shoalhaven \\ Cancer Care Centre, Shoalhaven Hospital, NSW, Australia \\ Contributions: (I) Conception and design: K Juszczyk, R Winn, S Kang, D Brungs; (II) Administrative support: K Juszczyk, S Kang, D Brungs; (III) \\ Provision of study materials or patients: R Winn, S Putnis, S Kang, J Chen, G Fylyk, M Aghmesheh, D Brungs; (IV) Collection and assembly of data: \\ S Kang; (V) Data analysis and interpretation: K Juszczyk, D Brungs; (VI) Manuscript writing: All authors; (VII) Final approval of manuscript: All \\ authors. \\ Correspondence to: Karolina Juszczyk. Division of Surgery, Colorectal Unit, Wollongong Hospital, Loftus St Wollongong, NSW 2500, Australia. \\ Email: karolina_juszczyk@hotmail.com.
}

Background: The impact of increased body mass index (BMI) on clinical outcomes in locoregional rectal cancer is unknown.

Methods: This is a retrospective cohort study which included 453 consecutive rectal cancer patients undergoing definitive treatment, with confirmed stage I, II or III rectal adenocarcinoma. The association of BMI at diagnosis with overall survival (OS), cancer specific survival (CSS) and disease-free survival (DFS) was explored, controlling for key covariates using multivariable analyses. BMI as defined by the World Health Organization (WHO) is as follows: BMI <18.5-underweight; 18.5-24.9—normal; 25.0-29.9-preobesity; >30_obese.

Results: Overweight and obese patients had significantly better OS than underweight/normal weight patients (5-year OS $80 \%$ for overweight, $77 \%$ for obese, and $65 \%$ for underweight/normal weight patients, $\mathrm{P}=0.02)$. High BMI (>25) was significantly associated with improved OS in univariate [0.62 (0.4-0.8) $\mathrm{P}=0.007]$ and multivariable $[0.65(0.4-0.9) \mathrm{P}=0.023]$ analyses. When stratified by stage, high $\mathrm{BMI}$ was associated with improved OS in stage III patients $(\mathrm{P}=0.0009)$, but not stage II $(\mathrm{P}=0.21)$ or stage I $(0.54)$. High $\mathrm{BMI}$ was also significantly associated with improved CSS in univariate (HR 0.62, $\mathrm{P}=0.048$ ) and multivariable analyses (HR 0.58, $\mathrm{P}=0.03$ ).

Conclusions: In our study a BMI greater than 25 is significantly associated with a longer OS and CSS in patients with locoregional rectal cancer. These findings may be due to the reduced metabolic capacity for non-obese patients to deal with rectal cancer treatment as well as the burden of disease, however further research is needed to evaluate this.

Keywords: Body mass index (BMI); cancer; obesity; survival; rectal cancer

Submitted Jan 29, 2020. Accepted for publication Jun 05, 2020.

doi: 10.21037/jgo-20-48

View this article at: http://dx.doi.org/10.21037/jgo-20-48 


\section{Introduction}

Colorectal cancer is a common and lethal malignancy, accounting for an estimated $12.3 \%$ of all new cancers diagnosed in 2018 and estimated $8.5 \%$ of all deaths from cancer in 2018 in Australia (1). Obesity, as reflected by a high body mass index (BMI), is a known risk factor for the development of colorectal cancer (2). It has also been shown to contribute to greater morbidity and short term postoperative complications in colorectal surgery (3). However, the impact of BMI on longer term clinical outcomes in locoregional rectal cancer is uncertain with conflicting results from published studies. While some surgical series demonstrate improved overall survival (OS) in overweight and obese patients compared with underweight patients (4-7), other studies have contrasting results with a decreased survival seen in overweight/obese patients $(8,9)$, or no difference in OS across BMI categories (10-14).

In Australia, as well as an increasing incidence of rectal cancer, we are also experiencing an increasing incidence of obesity $(15,16)$. A Queensland study (17) investigated the impact of weight on mortality using data from 1,825 patients diagnosed with stage I-III colorectal cancer. They demonstrated that overweight, but not obese patients, had an improved OS compared to those with a normal BMI. Underweight patients had a significantly higher mortality risk. They also found that excessive weight loss of five kilograms or more at any period was associated with increased all-cause and colorectal cancer-specific mortality.

We undertook this study to evaluate how BMI affects OS, cancer specific survival (CSS) and disease-free survival (DFS) in locoregional rectal cancer receiving curative treatment. We present the following article in accordance with the STROBE reporting checklist (available at http:// dx.doi.org/10.21037/jgo-20-48).

\section{Methods}

\section{Patient cohort}

Using electronic medical records and cancer registry data, we identified all patients with histopathological confirmed stage I, II or III rectal adenocarcinoma, undergoing definitive treatment, that were managed in the Illawarra Shoalhaven Local Health District (New South Wales, Australia) between 2006-2017. Staging was based on the 8th edition of the American Joint Committee on Cancer and College of American Joint Pathologists (AJCC) (18). Staging was based on clinical stage for patients undergoing neoadjuvant therapy, or pathological stage for patients who had surgery upfront. Patients managed with non-curative or palliative intent were excluded. A total of 453 patients managed with curative intent were included in the analysis, regardless of neoadjuvant or adjuvant treatments. For each patient the following was extracted from the medical record: age, gender, TNM stage, presence of lymphovascular (LVI) or perineural (PNI) invasion, histopathological grade, BMI, length of stay (LOS), type of surgery, pretreatment CEA, neoadjuvant/adjuvant radiotherapy and chemotherapy, and long term outcomes including OS, CSS, and DFS. BMI was collected at diagnosis of rectal cancer, and defined by the World Health Organization (WHO) is as follows: BMI $<18.5$-underweight, BMI 18.5-24.9-normal weight, BMI 25.0-29.9-pre-obesity, BMI >30—obese (19). BMI was collected at the time of diagnosis.

The research was conducted in accordance with the Declaration of Helsinki (as revised in 2013) lsinki. This study was approved by the NSW Population \& Health Services Research Ethics Committee (LNR/15/ WGONG/61).

\section{Statistical analysis}

Our primary outcome was impact of BMI on all cause OS. The secondary outcomes were CSS, DFS, and surgical complications measured by LOS. Patient characteristics were compared with ChiSq. Median values for OS, CSS, and DFS, and corresponding $95 \% \mathrm{CI}$ were calculated using Kaplan-Meier methods. Unadjusted and multivariable Cox proportional hazards regression analyses were used to estimate the association between BMI and survival outcomes, and to calculate corresponding hazard ratios (HRs) and 95\% confidence intervals (CIs). All variables significant in the univariate analysis $(\mathrm{P}<0.05)$ were included in the multivariable model. All statistical analyses were performed using SAS 9.2 software (SAS Institute, Inc., Cary, NC, USA).

\section{Results}

\section{Patient characteristics $(n=453)$}

The characteristics of the included patients are summarized in Table 1. The mean follow-up was 3.2 years. At the end of the follow-up period, 144 (32\%) patients had died, with 79 deaths (55\% of deaths) due to rectal cancer. One hundred and twenty-four $(27 \%)$ patients had local recurrence or 
Table 1 Patient characteristics $[\mathrm{n}=453]$

\begin{tabular}{|c|c|c|c|c|}
\hline Patient characteristics & All patients & $\begin{array}{l}\text { Underweight/normal weight } \\
\qquad(\mathrm{BMI} \leq 25), \mathrm{N}=124\end{array}$ & $\begin{array}{l}\text { Overweight /obese } \\
(\mathrm{BMI}>25), \mathrm{N}=329\end{array}$ & $P$ value \\
\hline \multicolumn{5}{|l|}{ Age } \\
\hline$<65$ & $171[38]$ & $50[40]$ & $121[37]$ & 0.05 \\
\hline $65-75$ & $151[33]$ & 31 [25] & $120[36]$ & \\
\hline Male sex & $297[66]$ & 74 [60] & $223[67]$ & 0.10 \\
\hline \multicolumn{5}{|l|}{ TNM stage } \\
\hline 1 & $124[27]$ & 28 [23] & 96 [29] & 0.28 \\
\hline II & $89[18]$ & $21[17]$ & $60[18]$ & \\
\hline PNI present ${ }^{\mathrm{b}}$ & 79 [18] & 25 [21] & $54[17]$ & 0.35 \\
\hline \multicolumn{5}{|l|}{ BMI } \\
\hline Underweight $(<18.5)$ & $4[1]$ & 4 & & \\
\hline Normal (18.5-25) & $120[26]$ & 120 & & \\
\hline Overweight [25-30] & $198[44]$ & & 198 & \\
\hline Obese $(>30)$ & $131[29]$ & & 131 & \\
\hline \multicolumn{5}{|l|}{ Pre-treatment CEA } \\
\hline Median length of stay (days) & 10 & 10 & 10 & \\
\hline
\end{tabular}

distal metastases from their disease. Four (1\%) patients were underweight, 120 (26\%) patients had a normal BMI, 198 (44\%) were overweight, and 131 (29\%) were obese, similar to reported demographics of the Australian population $(15,16)$. The weight groups had similar demographic and clinicopathological characteristics, apart from pre-treatment CEA which was less likely to be elevated in overweight and obese patients compared to underweight/normal weight patients ( $20 \%$ vs. $32 \%, \mathrm{P}=0.0079)$. There was no significant difference in use of laparoscopic surgery, neoadjuvant radiotherapy or adjuvant chemotherapy, or postoperative LOS between BMI groups (Table 1).

\section{BMI and survival outcomes}

Overweight and obese patients had significantly better OS than underweight/normal weight patients (5-year OS $80 \%$, $77 \%, 65 \%$ respectively, $\mathrm{P}=0.02)$. As there was no significant difference in survival outcomes between overweight and obese patients, these groups were combined for subsequent analyses. In univariate analysis, BMI $>25$ was associated with improved OS (HR 0.62; 95\% CI, 0.4-0.8, $\mathrm{P}=0.007$ ) (Table 2, Figure 1). In multivariable analysis, after adjusting for age, TNM stage, tumour grade, receipt of chemotherapy, and LVI, high BMI remained significantly associated with 
Table 2 Univariate and multivariate analysis for overall survival

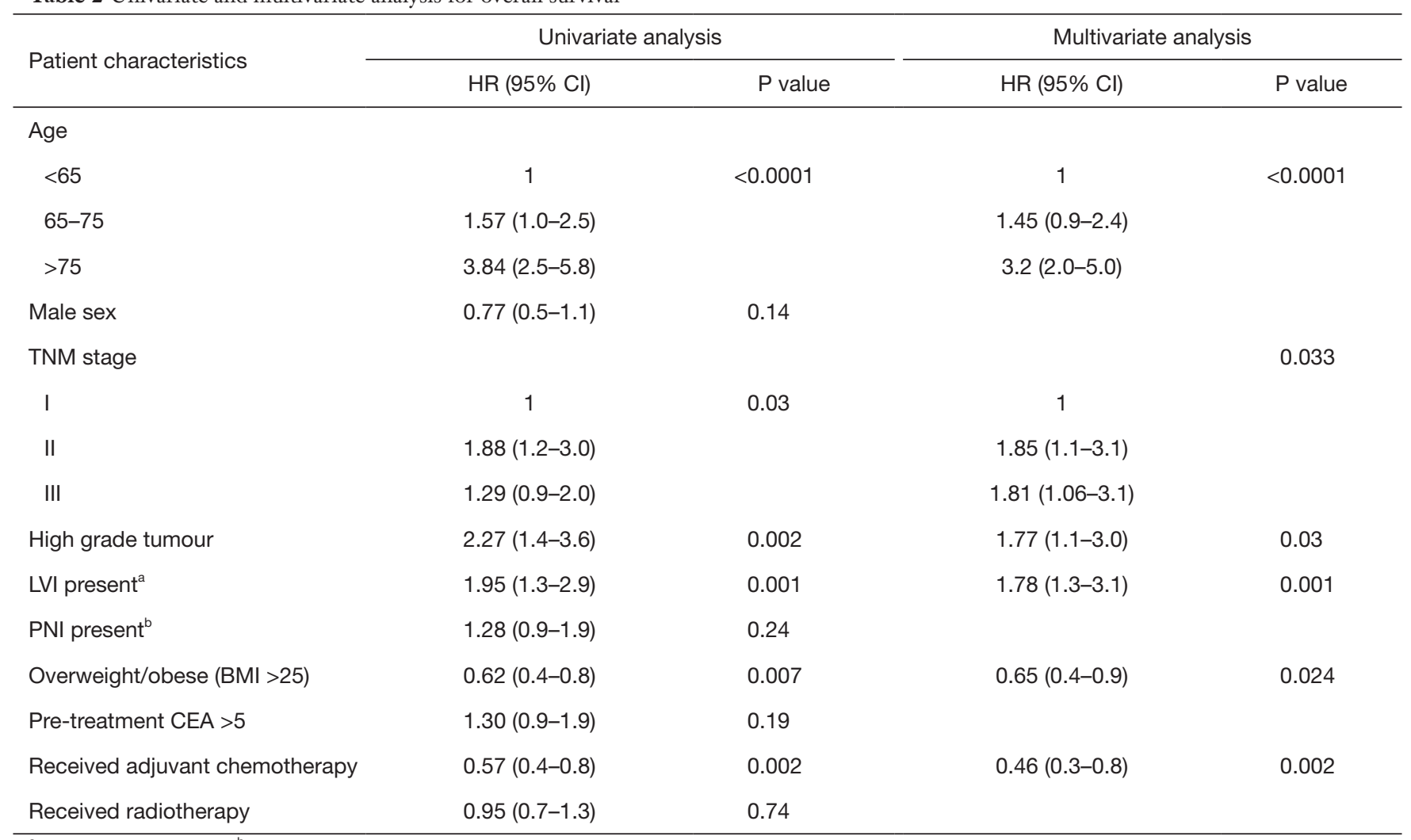

a , 27 results missing; ${ }^{\mathrm{b}}, 16$ results missing.

improved OS (HR 0.65; 95\% CI, 0.4-0.9, $\mathrm{P}=0.023$ ). When stratified by stage, high $\mathrm{BMI}$ was associated with improved OS in stage III patients ( $\mathrm{P}=0.0009)$, but not stage II $(\mathrm{P}=0.21)$ or stage I (0.54). High BMI (>25) was also significantly associated with CSS in univariate (HR 0.62; 95\% CI, 0.4$0.99, \mathrm{P}=0.048$ ), and multivariable analyses (HR $0.58 ; 95 \%$ CI, 0.3-0.98, $\mathrm{P}=0.03$ ) (Figure 1).

There was no significant association of BMI and DFS (HR 0.71; 95\% CI, 0.5-1.1, P=0.08) (Figure 1). Similarly, overweight and obese patients had no significant increased risk of local recurrence compared to normal/underweight patients $(6 \%$ vs. $7 \%, \mathrm{P}=0.64)$.

\section{Discussion}

The central finding of our study is that overweight and obese patients had significantly better OS than underweight/ normal weight patients (5-year OS $80 \%, 77 \%, 65 \%$ respectively). This association persisted after adjustment for known confounders on multivariable analyses. Similar results have been demonstrated by other studies in colorectal cancer. Shahjehan et al. (4) found underweight patients had poorer survival in patients with stage III and IV disease, while Ballian et al. (6) showed improved survival in obese rectal cancer patients. Similar results were seen by You et al. (7), who found the 5-year disease free survival rate was lower in the underweight patients and higher in the obese patients with upper rectal cancer.

However, there are conflicting results in the literature, with other studies finding no impact of BMI on CSS (12), OS $(13,14)$, or DFS $(13)$. One series examining patients with locoregional rectal cancer in Mexico showed an inferior DFS in obese patients, although these results may be due reduced utilization of neoadjuvant treatments in this group (8). In our study, clinicopathological characteristics and treatments were well matched between patient groups. We note a significantly lower number of patients with an elevated CEA in the overweight/obese patients despite similar TNM stage, a result which is likely due to the larger vascular volume and consequential haemodilution in obese patients (20).

In the current study, BMI appeared to have a stage 

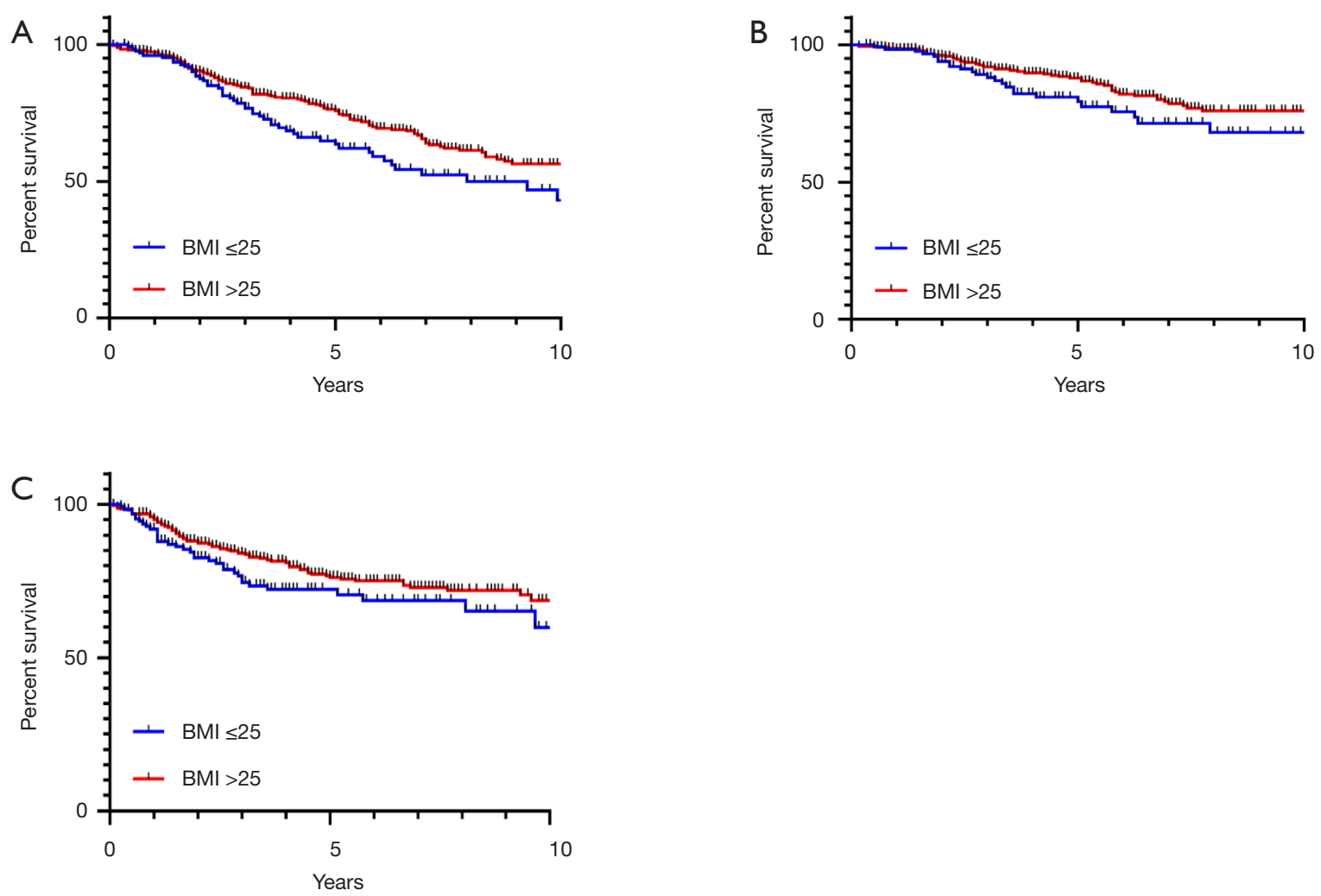

Figure 1 Association of BMI and (A) overall survival (B) cancer specific survival (C) disease free survival. BMI was associated with improved OS $(\mathrm{P}=0.007)$ and CSS $(\mathrm{P}=0.048)$ but not DFS $(0.08)$.

dependent effect, with improved OS in stage III patients ( $\mathrm{P}=0.0009)$, but not stage I or II. While this result is likely to be due, in part, to study power and event numbers, similar results have been reported by Kocarnik et al. (21), who found that all-cause mortality was higher in overweight patients in stage I, but lower in stage III and IV. It is postulated that this stage dependent effect is due to the reduced metabolic capacity of non-obese patients to cope with the more intensive treatment regimens and increased metabolic demands of advanced disease (22).

In addition to this concept of metabolic reserve, there are several other key factors that may explain the improved outcomes seen in patients with elevated BMIs. Very large multi-institutional surgical series have also shown improved survival in overweight and obese patients (23). This is thought to be driven by a chronic state of low-grade inflammation in overweight or mildly obese patients which allow a faster response to the stress of surgery (24). More intriguingly, hepatic steatosis due to obesity may actually protect against the establishment of metastases, with reduced rates of hepatic metastases seen in colorectal cancer patients with hepatic steatosis (25). There appears to be a complex interplay at the molecular level between factors affecting obesity, the immune system and oncogenesis which has not yet been fully established. Key areas for further research include elucidating underlying pathophysiological processes, studying the impact of weight change during treatments, and identifying more robust markers of nutrition in cancer patients.

There are several limitations in our study. This is a retrospective study and is limited by the biases inherent to this study design. There are likely to be additional unmeasured confounders, such as patient comorbidities, which have influenced the observed results. BMI is also a crude measure of obesity and other more precise measures may more accurately evaluate this relationship such as mesorectal fat area or sarcopenia $(26,27)$. BMI was also measured at diagnosis only, and we were unable to capture changes in BMI over time. Of particular importance, weight loss prior to diagnosis has been shown to be a poor prognostic factor, and is likely to have contributed to the inferior outcomes seen in normal weight and under weight 
patients (28). Lastly, there was only a small number of underweight patients limiting conclusions from this patient group.

\section{Conclusions}

$\mathrm{BMI}$ is a key consideration in outcomes for patients with locoregional rectal cancer.

\section{Acknowledgments}

Funding: None.

\section{Footnote}

Reporting Checklist: The authors have completed the STROBE reporting checklist. Available at http://dx.doi. org/10.21037/jgo-20-48

Conflicts of Interest: All authors have completed the ICMJE uniform disclosure form (available at http://dx.doi. org/10.21037/jgo-20-48). The authors have no conflicts of interest to declare.

Ethical Statement: The authors are accountable for all aspects of the work in ensuring that questions related to the accuracy or integrity of any part of the work are appropriately investigated and resolved. The research was conducted in accordance with the Declaration of Helsinki (as revised in 2013). This study was approved by the NSW Population \& Health Services Research Ethics Committee (LNR/15/WGONG/61).

Open Access Statement: This is an Open Access article distributed in accordance with the Creative Commons Attribution-NonCommercial-NoDerivs 4.0 International License (CC BY-NC-ND 4.0), which permits the noncommercial replication and distribution of the article with the strict proviso that no changes or edits are made and the original work is properly cited (including links to both the formal publication through the relevant DOI and the license). See: https://creativecommons.org/licenses/by-nc-nd/4.0/.

\section{References}

1. Cancer data in Australia, web report last updated 18/12/18. AIHW 2018. Available online: https://www.aihw.gov.au/ reports/cancer/cancer-data-in-australia/contents/summary
2. Bardou M, Barkun AN, Martel M. Obesity and colorectal cancer. Gut 2013;62:933-47.

3. Poelemeijer YQM, Lijftogt N, Detering R, et al. Obesity as a determinant of perioperative and postoperative outcome in patients following colorectal cancer surgery: A population-based study (2009-2016). Eur J Surg Oncol 2018;44:1849-57.

4. Shahjehan F, Merchea A, Cochuyt JJ, et al. Body mass index and long-term outcomes in patients with colorectal cancer. Front Oncol 2018;8:620.

5. Schlesinger S, Siegert S, Koch M, et al. Postdiagnosis body mass index and risk of mortality in colorectal cancer survivors: a prospective study and meta-analysis. Cancer Causes Control 2014;25:1407-18.

6. Ballian N, Yamane B, Leverson G, et al. Body mass index does not affect postoperative morbidity and oncologic outcomes of total mesorectal excision for rectal adenocarninoma. Ann Surg Oncol 2010;17:1606-13.

7. You J, Tang R, Changchien C, et al. Effect of body mass index on the outcome of patients with rectal cancer receiving curative anterior resection. Ann Surg 2009;249:783-7.

8. Lino-Silva LS, Aguilar-Cruz E, Salcedo-Hernández RA, et al. Overweight but not obesity is associated with decreased survival in rectal cancer. Contemp Oncol (Pozn) 2018;22:158-64.

9. Campbell PT, Newton CC, Newcomb PA, et al. Association between body mass index and mortality for colorectal cancer survivors: overall and by tumor molecular phenotype. Cancer Epidemiol Biomarkers Prev 2015;24:1229-38.

10. De Felice F, Musio D, Magnante AL, et al. Excess of weight: is it a modifiable predictive and prognostic factor in locally advanced rectal cancer? Eur Rev Med Pharmacol Sci 2017;21:4606-12.

11. Maskarinec G, Harmon BE, Little MA, et al. Excess body weight and colorectal cancer survival: the multiethnic cohort. Cancer Causes Control 2015;26:1709-18.

12. Meyerhardt JA, Niedzwiecki D, Hoolis D, et al. Impact of body mass index and weight change after treatment on cancer recurrence and survival in patients with stage III colon cancer: findings from cancer and leukemia group B 89803. J Clin Oncol 2008;26:4109-15.

13. Denost Q, Quintane L, Buscail E, et al. Short and longterm impact of body mass index on laparoscopic rectal cancer surgery. Colorectal Dis 2013;15:463-9.

14. Sun $Y, X u Z$, Lin $H$, et al. Impact of body mass index on treatment outcome of neoadjuvant chemoradiotherapy 
in locally advanced rectal cancer. Eur J Surg Oncol 2017;43:1828-34.

15. An interactive insight into overweight and obesity in Australia. Nov 2017. Australian Institute of Health and Welfare.

16. Cancer in Australia 2019. Australian Institute of Health and Welfare.

17. Baade PD, Meng X, Youl PH, et al. The impact of body mass index and physical activity on mortality among patients with colorectal cancer in Queensland, Australia. Cancer Epidemiol Biomarkers Prev 2011;20:1410-20.

18. Amin MB, Edge SB, Greene FL et al. AJCC Cancer Staging Manual 8th Edit. 2017.

19. BMI Classification (accessed 29/4/19) World Health Organization. Available online: http://www.euro.who.int/ en/health-topics/disease-prevention/nutrition/a-healthylifestyle/body-mass-index-bmi

20. Chen W, Liu Q, Tan SY, et al. Association between carcinoembryonic antigen, carbohydrate antigen 19-9 and body mass index in colorectal cancer patients. Mol Clin Oncol 2013;1:879-86.

21. Kocarnik JM, Chan AT, Slattery ML, et al. Relationship of pre-diagnostic body mass index with survival after colorectal cancer: stage-specific associations. Int J Cancer 2016;139:1065-72.

22. Kasi PM, Zafar SY, Grothey A. Is obesity an advantage in

Cite this article as: Juszczyk K, Kang S, Putnis S, Winn R, Chen J, Aghmesheh M, Fylyk G, Brungs D. High body mass index is associated with an increased overall survival in rectal cancer. J Gastrointest Oncol 2020;11(4):626-632. doi: 10.21037/ jgo-20-48 patients with colorectal cancer? Expert Rev Gastroenterol Hepatol 2015;9:1339-42.

23. Mullen JT, Moorman DW, Davenport DL. The obesity paradox: body mass index and outcomes in patients undergoing nonbariatric general surgery. Ann Surg 2009;250:166-72.

24. Gil A, María Aguilera C, Gil-Campos M, et al. Altered signalling and gene expression associated with the immune system and the inflammatory response in obesity. Br J Nutr 2007;98 Suppl 1:S121-6.

25. Murono K, Kitayama J, Tsuno NH, et al. Hepatic steatosis is associated with lower incidence of liver metastasis from colorectal cancer. Int J Colorectal Dis 2013;28:1065-72.

26. Yoon J, Chung YE, Lim JS, et al. Quantitative assessment of mesorectal fat: new prognostic biomarker in patients with mid-to-lower rectal cancer. Eur Radiol 2019;29:1240-7.

27. Jochum SB, Kistner M, Wood EH, et al. Is sarcopenia a better predictor of complications than body mass index? Sarcopenia and surgical outcomes in patients with rectal cancer. Colorectal Dis 2019;21:1372-8.

28. Walter V, Jansen L, Hoffmeister M, et al. Prognostic relevance of prediagnostic weight loss and overweight at diagnosis in patients with colorectal cancer. Am J Clin Nutr 2016;104:1110-20. 